\title{
Invasive Fungal Infections in Children with Acute Lymphoblastic Leukemia: Experience from a Reference University Hospital in Cappadocia
}

\author{
Ebru YILMAZ1, Arda ERDOGMUS ${ }^{1,2}$, Alper OZCAN ${ }^{1}$, Sureyya Burcu GORKEM ${ }^{3}$, \\ Ozgur CEYLAN ${ }^{4,5}$, Kemal DENIZ ${ }^{6}$, Ekrem UNAL ${ }^{1,7}$, Mustafa Altay ATALAY ${ }^{2}$, \\ Musa KARAKUKCU ${ }^{1}$, Ayse Nedret KOC ${ }^{2}$, Turkan PATIROGLU ${ }^{1}$
}

\footnotetext{
${ }^{1}$ Erciyes University, Faculty of Medicine, Department of Pediatrics, Division of Pediatric Hematology Oncology, Kayseri

${ }^{2}$ Erciyes University, Faculty of Medicine, Department of Medical Microbiology, Kayseri

${ }^{3}$ Erciyes University, Faculty of Medicine, Department of Radiology, Division of Pediatric Radiology, Kayseri

${ }^{4}$ Erciyes University, Faculty of Medicine, Department of Pediatrics, Division of Pediatric Infectious Disease, Kayseri

${ }^{5}$ Baskent University, Faculty of Medicine, Department of Pediatrics, Division of Pediatric Infectious Disease, Adana

${ }^{6}$ Erciyes University, Faculty of Medicine, Department of Pathology, Kayseri

${ }^{7}$ Erciyes University, Gevher Nesibe Genom and Stem Cell Institution, Genome and Stem Cell Center (GENKOK), Department of Molecular Biology and Genetic, Kayseri, TURKEY
}

\begin{abstract}
Invasive fungal infections (IFI) are an important cause of mortality and morbidity in patients with hematological malignancy. This study aims to investigate the incidence of IFI development, risk factors, the management of the infection in a pediatric patient group followed up with the diagnosis of acute lymphoblastic leukemia (ALL), and to share the experience obtained from a single center. Two hundred forty children monitored with the diagnosis of ALL in the pediatric hematology-oncology department of the Erciyes University Medical Faculty from January 2010 to September 2017 included in the study. A total of 30 (14 females and 14 males) IFI attacks were diagnosed (12.5\%) in the included patients with ALL, two of them having the attacks twice. Candida species were the dominant cause of infection $(n=17)$ and the rest $(n=8)$ had invasive Aspergillosis. Nineteen IFI attacks were assessed as proven, 6 as probable, and 5 as possible IFI. The most fungal infection was detected in blood culture (43.3\%) followed by pulmonary involvement (40\%). The most frequently used diagnostic methods were direct microscopic examination, histological examination, and cultures (66.6\%). IFI-related mortality was 20\%. IFI continues to be an important problem in pediatric patients with hematologic malignity. The 7 of the observed invasive Aspergillosis developed in non hepafiltered room. Treatment of neutropenic children in hepafiltered rooms decrease the risk of IFI. With careful assessment of the patients bearing risk factors for IFI development, early diagnosis and treatment will reduce morbidity and mortality.
\end{abstract}

Keywords: Leukemia, Children, Invasive fungal infections

\section{INTRODUCTION}

Invasive fungal infections (IFI) is an important cause of mortality and morbidity in patients with hematological malignancies. Approximately $5-10 \%$ of febrile neutropenia attacks results from proven yeast or mould infections. ${ }^{1}$ The most important reported risk factors of IFI in children with acute lymphoblastic leukemia (ALL) are the us- age of steroids, deep and prolonged neutropenia due to the intensive chemotherapy, prolonged usage of broad spectrum antibiotics, central venous catheters, total parenteral nutrition (TPN), severe mucositis. ${ }^{2-5}$ Although early diagnosis and intervention reduce the IFI-related mortality, the use of diagnostic methods in children with ALL are limited due to their invasive characteristics. 
This study aims to investigate the incidence, risk factors, the management of IFI in children with ALL.

\section{PATIENTS and METHODS}

Access was gained retrospectively to the data belonging to the patients monitored with the diagnosis of ALL in the pediatric hematology-oncology department of the Erciyes University Medical Faculty from January 2010 to September 2017. Included in the study were the patients who were diagnosed, through clinical, laboratory, and imaging methods, to have proven, probable, and possible IFI according to the criteria of the European Organization for Research and Treatment of Cancer and Mycoses Study Group (EORTC/MSG). ${ }^{6}$ Patients diagnosed with Pneumocystis jirovecii were excluded from the study. The data transferred from the patients' files and the hospital's database and recorded into their standard forms included the patients' ages, sexes, the risk groups according to Turkish ALL (TRALL) 2000, ALL IC BerlinFrankfurt-Münster (BFM) 2009 and Interfant 2006 protocols, the results of the laboratory and imaging methods employed used for diagnosis and followup of IFI, the development period of IFI, the treatment given and its duration, the symptoms emerging during the development period of IFI, absolute neutrophil count (ANC), absence or presence of central venous catheter, feeding with TPN, the state of being monitored in a single room with hepafilter, the use of granulocyte colony-stimulating factor (GCSF) and granulocyte suspension, and IFI reactivation. ${ }^{7,8}$ Ethical permission for a review of all records was granted by the ethics committee of Erciyes University (Approval Number: 2018/552, Date: 07.11.2018).

\section{Statistical Analyses}

The descriptive statistics and quantitative variables using Shapiro-Wilk test were expressed as mean \pm standard deviation or median (minimum-maximum) according to whether the distributions were normal or not. Nominal variables, however, were expressed as the number of cases and percentage $(\%)$.

\begin{tabular}{|ll|}
\hline $\begin{array}{l}\text { Table 1. Characteristics of } 240 \text { patients with acute lymphoid } \\
\text { leukemia }\end{array}$ \\
\hline Characteristic & $\mathbf{n}$ \\
\hline Age (years), median & $6(0,4-18)$ \\
Gender male/female & $141 / 99$ \\
Risk group & \\
SRG & $45(18.75 \%)$ \\
MRG & $118(49.1 \%)$ \\
HRG & $27(11.25 \%)$ \\
Relaps leukemia & $44(18.3 \%)$ \\
Refractory leukemia & $6(2.5 \%)$ \\
\hline SRG= Standard Risk Group, MRG= Medium Risk Group, \\
HRG= High Risk Group
\end{tabular}

\section{RESULTS}

Access was achieved to the data regarding 240 patients (99 female and 141 male) monitored with the diagnosis of ALL. According to TRALL 2000, ALLIC BFM 2009, interfant 2006 treatment protocols, 45 of the patients were classified as standard risk group (SRG), 118 were medium risk group (MRG), 27 high risk group (HRG) and 44/6 were relaps/refractory leukemia. The baseline characteristics of the ALL patients are shown in Table 1.

With IFI detected twice at different times in 2 patients, a total of 30 (14 female and 14 male) IFI attacks were diagnosed. The median age of the patients was 93 months (14-212 months). The characteristics of the 28 patients diagnosed with IFI are shown in Table 2.

While Aspergillus niger was isolated in brain biopsy of one patient that was previously published. ${ }^{9,10}$ Aspergillus spp. was isolated in pleural fluid cultures of two patients. Five patients, owing to their general condition being poor, were diagnosed to have Aspergillus spp based on serial galactomannan and imaging results. One patient had mucormycosis, one patient had Geotrichum capitatum, 4 patients had Candida albicans, and 10 patients had non-albicans Candida. While in one of the 2 patients with fungal proliferation at different times, C. glabrata and Acremonium strictum proliferated, in the other, C. glabrata and C. pelliculosa was generated. 


\begin{tabular}{|c|c|}
\hline Characteristic & $\mathbf{n}$ \\
\hline Age (months), median & $93(14-212)$ \\
\hline Gender male/female & $14 / 14$ \\
\hline \multicolumn{2}{|l|}{ Risk group } \\
\hline SRG & $2(7.14 \%)$ \\
\hline MRG & $8(28.57 \%)$ \\
\hline $\mathrm{HRG}$ & $8(28.57 \%)$ \\
\hline Relaps & $7(25 \%)$ \\
\hline Refractory leukemia & $3(10.71 \%)$ \\
\hline \multicolumn{2}{|l|}{ Treatment phase } \\
\hline Induction & $8(26.6 \%)$ \\
\hline Reinduction & $2(6.6 \%)$ \\
\hline \multicolumn{2}{|c|}{ Consolidation (HR blocks, protocol M) 10 (33.3\%) } \\
\hline Maintenance therapy & $1(3.3 \%)$ \\
\hline R blocks of REZ BFM 2002 & $3(10 \%)$ \\
\hline FLAG protocol & $4(13.3 \%)$ \\
\hline TVTC protocol & $2(6.6 \%)$ \\
\hline \multicolumn{2}{|l|}{ Infection type } \\
\hline Proven & 19 (63.3\%) \\
\hline Probable & $6(20 \%)$ \\
\hline Possible & $5(16.6 \%)$ \\
\hline \multicolumn{2}{|l|}{ Presence of symptoms } \\
\hline Fever & 29 (96.6\%) \\
\hline Cough & $8(26.6 \%)$ \\
\hline Dyspnea & $3(10 \%)$ \\
\hline Focal neurological finding & $1(3.3 \%)$ \\
\hline Nasal defect & 1 (3.3\%) \\
\hline Abdominal pain & 2 (6.6\%) \\
\hline \multicolumn{2}{|c|}{$\begin{array}{l}\text { SRG= Standard Risk Group, MRG= Medium Risk Group, } \\
\text { HRG= High Risk Group, FLAG= fludarabine, high-dose cytarabine, } \\
\text { G-CSF, TVTC= Topotecan, Vinorelbine, Thiotepa, Clofarabine }\end{array}$} \\
\hline
\end{tabular}

Monotherapy (L-AmB, caspofungin, fluconazole or variconazole) was applied to 15 IFI attacks and combined therapy was applied to 15 IFI. Median duration of treatment 30 (21-720) days. In addition to antifungal therapy, 12 patients received granulocyte suspension (3 patients in addition to monotherapy and 9 patients in addition to combined therapy). The patients other than those in induction period and relapsing/refractory ones received 5 $\mathrm{mcg} / \mathrm{kg} /$ day GCSF.

\begin{tabular}{|c|c|}
\hline Risk Factors & n (\%) \\
\hline Steroid therapy (>14 days) & $10(33.3 \%)$ \\
\hline Severe neutropenia ( $>10$ days) & $30(100 \%)$ \\
\hline Antibiotic use (>7 days) & $30(100 \%)$ \\
\hline Total parenteral nutrition & $15(50 \%)$ \\
\hline Central venous line & 29 (96.6\%) \\
\hline
\end{tabular}

Nineteen IFI attacks were assessed as proven, 6 as probable, and 5 as possible IFI. The most fungal infection was detected blood culture $(43.3 \%)$ followed by in the lungs (40\%). The most frequent symptom in 30 IFI attacks was fever $(96.6 \%)$, and the most frequently employed diagnostic method was conventional mycological examination (direct microscopic examination, histological examination, and culture) (66.6\%).

Median ANC was $100 / \mathrm{mm}^{3}\left(0-330 \mathrm{~mm}^{3}\right)$ and the period when ANC remained less than 500 was 45 days (11-109 days). All of the patients were taking broad spectrum antibiotics that last longer than 7 days. It was detected than only one of 8 patients diagnosed as having Aspergillus diagnosis and assessed as proven/probable IFI was hospitalized in the room with hepafilter. Five of the 14 IFI attacks with $C$. albicans/non-albicans Candida, 8 IFI attacks with invasive Aspergillus and 2 IFI attacks with proliferated G. capitatum, A. strictum detected had a history of TPN use. All the patients with IFI had central venous catheter, except the one patient in whom C. kefyr was grew from blood culture. The risk factors for developing invasive fungal infection are summarized in Table 3.

IFI deterioration occurred in 6 patients despite antifungal therapy, and 3 patients died of isolated fungal sepsis while 3 patients died of leukemia and fungal sepsis. Of the patients who died, 1 had been diagnosed as possible, 2 as probable, and 3 as proven IFI. Of our patients, 8 died of refractory leukemia although they had recovered completely with antifungal therapy (Overall mortality was $46.6 \%$ ). 


\section{DISCUSSION}

Leukemia ranks the first among the cancers of pediatric period, and acute leukemia constitutes more than $95 \%$ of leukemic cases, and acute lymphoblastic cases constitute $75-80 \%$ of acute leukemia cases. ${ }^{11}$ In recent years, considerable progress has been made in the treatment of pediatric ALL, and the 5-year survival rate is over $\% 85$. Relapse occurs in nearly $20 \%$ of ALL cases, and the prognosis of these cases is poor. ${ }^{12}$ In our study it has been determined that, consistent with the literature, $10 \%$ of our patients ( 25 of 240 patients) received the diagnosis of T-ALL, and the rate of relapse was $18,3 \%$.

The incidence of IFI has recently increased in parallel with the use of high dose chemotherapy. ${ }^{13}$ Rosen et al. ${ }^{14}$ have reported the IFI incidence to be $4.6 \%$ in pediatric patients monitored for solid tumor and leukemia, and deprived of primary antifungal prophylaxis. Mor et al. ${ }^{15}$, on the other hand, while reporting the IFI incidence in pediatric patients with hematologic disease and solid tumor to be $7.2 \%$, in the same study found the incidence in AML patients to be \%39.4. In ALL patients, however, it was established to be $11.2 \%$. In a study including 125 pediatric patients diagnosed to have ALL, the incidence of IFI has been reported to be $19.2 \%$. In our study it was $12.5 \%$.

Long-term, severe neutropenia, steroid use, central venous catheter use, mucositis associated with high dosed methotrexate use are reported to be the risk factors in IFI development. ${ }^{2-4}$ In addition, in patients with hematologic malignity, even if their ANC is normal functional neutropenia occurs, which phagocytosis and pathogens being destroyed. ${ }^{16}$ Observed in all of our patients were severe neutropenia lasting longer than 10 days, the use of central venous catheter in 29 IFI attacks, the use of TPN in 15 IFI attacks. Ten patients had to receive steroid longer than 14 days as required by the induction/re-induction protocols.

The most common observed IFI was Candida species $(46.6 \%)$ in our patients as previously reported. ${ }^{17,18}$ In the study by Baytan et al. ${ }^{19}$ which included 160 children with hematologic disease, IA was reported by $74 \%$ as the most common IFI agent, and hospital renovation activities are reported to have increased the risk. Some studies have argued that IA is correlated to contaminated ventilation system, hospital construction and renovation. ${ }^{19}$ In our study, it was found that IA developed in 1 and 7 of the 8 patients hospitalized with and without hepafilters, respectively.

In the study by Kaya et al. ${ }^{18}$ C. albicans have been found more frequently than non-albicans Candida species. However, in our study C. albicans have been detected in $5(35.7 \%)$ and non-albicans Candida species in $9(64.28 \%)$ of 14 patients, consistent with the studies supporting the opposite of the detection in the study of Kaya et al respectively. ${ }^{15,16}$

The use of histopathologic and/or cytopathologic diagnostic methods are restricted because of their invasive characteristics. Compared with pulmonary graph, High-resolution computed tomography (HRCT) has recently been regarded as a more valuable diagnostic method. ${ }^{20}$ The data obtained through radiological means in diagnosing IFI have a greater predictive value in pediatric patients, but they are not specific. ${ }^{21}$ The most frequently used diagnostic method for the 30 IFI attacks in our study has been conventional mycological examination $(66.6 \%)$ followed by computed tomography/ HRCT (30\%). A recent retrospective analysis of 139 pediatric invasive aspergillosis cases reported that the most frequent diagnostic radiologic finding was nodules at a rate of $34.6 \% .{ }^{22}$ Nodular appearance has been detected with HRCT in all of our patients.

Follow-up with galactomannan can contribute to early diagnosis and the improvement of clinical findings. However, its sensitivity has been reported to be $64.5 \%$ in diagnosing proven IA, $26.4 \%$ in probable IA, and $25.5 \%$ in possible IA. Herbert et al. ${ }^{23}$, have reported its specificity as $92.8 \%$ in adults and $47.6 \%$ in children. In the same study, the false positivity rate has been found to be in children while it is $0.9 \%$ in adults. ${ }^{23}$ Therefore, they should be used together with other diagnostic tools, and repetitive tests should be done. In our study it has been detected that while galactomannan positivity had been observed in 6 of the 8 patients diagnosed to have invasive aspergillus, and the diagnosis had been made histopathologically and without checking the galactomannan levels in two patients. 
In the study by Kaya et al. ${ }^{18}$ in which 154 pediatric patients with acute leukemia have been reported and fluconazole has been used as prophylaxis, the incidence has been found to be $13.6 \%$. In our study no patient has received any systemic antifungal prophylaxis other than nystatin used for oral care, and the IFI incidence has been found to be $12.5 \%$.

In studies on the recipients of hematopoietic stem cell transplantation and on the patients with hematologic malignancy, secondary antifungal prophylaxis has been found to be effective in preventing IFI from relapsing. However, there are no specific suggestions as to the drugs to be used for secondary prophylaxis. Nevertheless, there are publications suggesting that the pathogen causing the previous IFI and the response to previous antifungal therapy be considered when choosing the medicine. ${ }^{24-27}$ In the study by Liu et al. ${ }^{25}$ on 164 patients, however, no statistically significant difference in the recurrence rate has been obtained by using drugs and other broad-spectrum antifungal which has been used previously and proven to be effective. Secondary prophylaxis was not administered to patients other than who developed fungemia during the intensive chemotherapy period after complete recovery has been achieved with radiologic fungal antigen and/ or culture results. Despite this, IFI recurrence associated with the same factor was not observed in any of them. In 8 patients, however, antifungal therapy on end together with chemotherapy was continued until complete remission was achieved. With small number of the patients having been low and kept on antifungal and chemical therapy until complete remission, it would not be right to comment on secondary prophylaxis in the light of this study.

In empirical treatment, caspofungin and L-AmB are the first-line agents. ${ }^{4,25} \mathrm{In}$ our center L-AmB has been used for the first-line agent in empirical treatment. The antifungal drug used most frequently by our patients in our hospital has been L-AmB $(73.3 \%)$, and it has been used as monotherapy in 9 IFI attacks, and as adjuvant drug in combined therapy in 13 IFI attacks.

The rate of response to voriconazole in cancer patients is over $45 \% .{ }^{28}$ In our study voriconazol has been used as monotherapy in 5, and as part of a combined therapy in 13 of 18 IFI attacks. The com- bination of L-AmB and voriconazole was used in 11 patients, L-AmB and caspofungin in 2 patient, and combined voriconazole and caspofungin in 2 patients.

Mortality rate is still high despite advances in new antifungal drugs and supportive care. The IFI-related mortality in pediatric patients with hematologic malignity and aplastic anemia is in $40 \%$ range. $^{19}$ Despite the appropriate treatment, recurrence was observed by $16 \%$ following intensive chemotherapy. ${ }^{30}$ Recurrence was observed in none of our patients. However in 6 patients progression was observed, despite combined antifungal therapy, and three of the patients died of isolated fungal sepsis, and the other three died of refractory leukemia and IFI (IFI-related mortality was 20\%).

In this retrospective study, the data and experience that belong to a single center have been shared. Accordingly, there is a need for more comprehensive prospective randomized controlled studies so that risk factors (such as mucositis and neutropenia), risk specific fungal agents and treatment modalities (such as combined therapy, primary and secondary prophylaxis) can be identified.

IFI continues to be an important problem in pediatric patients with hematologic malignity. With careful assessment of the patients bearing risk factors for IFI development, early diagnosis and treatment will reduce morbidity and mortality. New diagnostic methods and antifungal treatment choices will contribute to the enhancement of the survival rate in this patient group.

\section{Acknowledgements:}

The authors thank to Prof. Dr. Mustafa Kursad OZTURK, Prof. Dr. Mehmet Akif OZDEMIR for their valuable contributions to the study.

\section{REFERENCES}

1. Karthaus M, Cornely OA. Recent developments in the management of invasive fungal infections in patients with hematological malignancies. Ann Hematol 84: 207-216, 2005

2. Patiroglu T, Altuner Torun $Y$, Yikilmaz A, et al. Invasive Pulmonary Aspergillosis Associated With Pleural Effusion and Pneumothorax. Erciyes Med J 30: 48-51, 2008. 
3. Celkan T, Kizilocak H, Evim M, et al. Hepatosplenic Fungal Infections in Children With Leukemia-Risk Factors and Outcome: A Multicentric Study. J Pediatr Hematol Oncol 41: 256-260, 2019

4. Lehrnbecher T, Phillips R, Alexander S, et al. Guideline for the management of fever and neutropenia in children with cancer and/or undergoing hematopoietic stem-cell transplantation. J Clin Oncol 30: 4427-4438, 2012.

5. Rüping MJ, Vehreschild JJ, Cornely OA. Patients at high risk of invasive fungal infections: when and how to treat. Drugs. 68: 1941-1962, 2008.

6. Donnelly JP, Chen SC, Kauffman CA, et al. Revision and Update of the Consensus Definitions of Invasive Fungal Disease From the European Organization for Research and Treatment of Cancer and the Mycoses Study Group Education and Research Consortium. Clin Infect Dis 71: 1367-1376, 2020.

7. Yuksel Soycan L. BFM-TR ALL 2000: first Turkish multicentric study in the treatment of pediatric acute lymphoblastic leukemia. J Pediatr Hematol Oncol 29: 21, 2007.

8. Uderzo C, Dini G, Locatelli F, et al. Treatment of childhood acute lymphoblastic leukemia after the first relapse: curative strategies. Haematologica 85: 47-53, 2000.

9. Patiroglu T, Unal E, Karakukcu M, et al. Multiple fungal brain abscesses in a child with acute lymphoblastic leukemia. Mycopathologia 174: 505-509, 2012.

10. Canpolat $\mathrm{M}$, Ceylan $\mathrm{O}$, Per $\mathrm{H}$, et al. Brain abscesses in children: results of 24 children from a reference center in Central Anatolia, Turkey. J Child Neurol 30: 458-467, 2015.

11. Arora RS, Arora B. Acute leukemia in children: A review of the current Indian data. South Asian J Cancer 5: 155-160, 2016.

12. Nguyen K, Devidas M, Cheng SC, et al. Factors influencing survival after relapse from acute lymphoblastic leukemia: a Children's Oncology Group study. Leukemia 22: 2142-2150, 2008.

13. Auberger J, Lass-Flörl C, Ulmer H, et al. Significant alterations in the epidemiology and treatment outcome of invasive fungal infections in patients with hematological malignancies. Int $\mathrm{J}$ Hematol 88: 508-515, 2008.

14. Rosen GP, Nielsen K, Glenn S, et al. Invasive fungal infections in pediatric oncology patients: 11-year experience at a single institution. J Pediatr Hematol Oncol 27: 135-140, 2005.

15. Mor M, Gilad G, Kornreich L, et al. Invasive fungal infections in pediatric oncology. Pediatr Blood Cancer 56: 1092-1097, 2011.

16. Sahbudak Bal Z, Yilmaz Karapinar D, Karadas N, et al. Proven and probable invasive fungal infections in children with acute lymphoblastic leukaemia: results from an university hospital, 2005-2013. Mycoses 58: 225-232, 2015.

17. Hazar V, Karasu GT, Uygun V, et al. Risks and outcomes of invasive fungal infections in pediatric allogeneic hematopoietic stem cell transplant recipients receiving fluconazole prophylaxis: a multicenter cohort study by the Turkish Pedi- atric Bone Marrow Transplantation Study Group. Med Mycol 57: 161-170, 2019.

18. Kaya Z, Gursel T, Kocak U, et al. Invasive fungal infections in pediatric leukemia patients receiving fluconazole prophylaxis. Pediatr Blood Cancer 52: 470-475, 2009.

19. Baytan B, Gunes AM, Celebi S, Gunay Ü. Invasive fungal diseases in children with hematologic disorders. Turk J Haematol 26: 190-196, 2009.

20. Althoff Souza C, Müller NL, Marchiori E, et al. Pulmonary invasive aspergillosis and candidiasis in immunocompromised patients: a comparative study of the high-resolution CT findings. J Thorac Imaging 21: 184-189, 2006.

21. Thomas KE, Owens CM, Veys PA, et al. The radiological spectrum of invasive aspergillosis in children: a 10-year review. Pediatr Radiol 33: 453-460, 2003.

22. Burgos A, Zaoutis TE, Dvorak CC, et al. Pediatric invasive aspergillosis: a multicenter retrospective analysis of 139 contemporary cases. Pediatrics 121: e1286-e1294, 2008.

23. Herbrecht R, Letscher-Bru V, Oprea C, et al. Aspergillus galactomannan detection in the diagnosis of invasive aspergillosis in cancer patients. J Clin Oncol 20: 1898-1906, 2002.

24. Sav H, Atalay MA, Koc AN, et al. Utility of the Aspergillus galactomannan antigen testing for neutropenic paediatric patients. Infez Med. 25: 38-44, 2017.

25. Tragiannidis A, Dokos C, Lehrnbecher T, Groll AH. Antifungal chemoprophylaxis in children and adolescents with haematological malignancies and following allogeneic haematopoietic stem cell transplantation: review of the literature and options for clinical practice. Drugs 72: 685-704, 2012.

26. Liu M, Li Y, Zhang $Y$, et al. Secondary antifungal prophylaxis in hematological malignancy patients with previous invasive fungal disease: a retrospective analysis. PLoS One 9: e115461, 2014

27. Lee JY, Jung CW, Kim K, Jang JH. Impact of previous invasive pulmonary aspergillosis on the outcome of allogeneic hematopoietic stem cell transplantation. Korean J Hematol 47: 255-259, 2012.

28. Walsh TJ, Lutsar I, Driscoll T, et al. Voriconazole in the treatment of aspergillosis, scedosporiosis and other invasive fungal infections in children. Pediatr Infect Dis J 21: 240-248, 2002.

29. Grigull L, Beier R, Schrauder A, et al. Invasive fungal infections are responsible for one-fifth of the infectious deaths in children with ALL. Mycoses 46: 441-446, 2003.

30. Cornely OA, Böhme A, Reichert D, et al. Risk factors for breakthrough invasive fungal infection during secondary prophylaxis. J Antimicrob Chemother 61: 939-946, 2008. 


\section{Correspondence:}

\section{Dr. Ekrem UNAL}

Erciyes Üniversitesi, Tip Fakültesi, Pediatri Anabilim Dali

Pediatri Hematoloji-Onkoloji ve Hematopoietik Kök Hücre

Nakli Merkezi

Moleküler Biyoloji ve Genetik Anabilim Dali

Gevher Nesibe Genom ve Kök Hucre Enstitusu

Genom ve Kök Hücre Merkezi

38010 Melikgazi, KAYSERI / TURKEY

Tel: (+90-352) 2076666 ext 25475

Fax: (+90-352) 4375825

e-mail: drekremunal@yahoo.com.tr

ekremunal@erciyes.edu.tr

\section{ORCIDs:}

Ebru Yilmaz

0000-0003-4802-0986

Arda Erdogmus

0000-0003-1895-9132

Alper Ozcan

0000-0002-6100-1205

Sureyya Burcu Gorkem

0000-0001-8949-6476

Ozgur Ceylan

0000-0001-6910-7250

Kemal Deniz

0000-0001-7749-2152

Ekrem Una

$0000-0002-2691-4826$

Mustafa Altay Atalay

Musa Karakukcu

0000-0003-4169-0637

Ayse Nedret Koc

0000-0003-2015-3541

Turkan Patiroglu

$0000-0002-1736-9707$

0000-0003-2471-764X 